Tropical Journal of Pharmaceutical Research, March 2008; 7 (1): 887-896

(C) Pharmacotherapy Group, Faculty of Pharmacy, University of Benin Benin City, Nigeria.

All rights reserved.

Research Article

Available online at http://www.tjpr.org

\title{
Physical and Release Properties of Metronidazole Suppositories
}

\author{
TA Adegboye and OA Itiola*
}

Department of Pharmaceutics \& Industrial Pharmacy, Faculty of Pharmacy, University of Ibadan, Ibadan, Nigeria

\begin{abstract}
Purpose: A study was made of the effects of some bases and adjuvants on the physical and release properties of metronidazole suppositories with a view to providing more information for the optimization of the rectal formulation of metronidazole.

Method: Suppositories (1g) containing 200mg of metronidazole each were prepared in witepsol (H15 and E75) and polyethylene glycol (PEG 2850 and 4650) bases, using different concentrations of Tween 80 , sodium salicylate and methylcellulose as adjuvants. The setting time, solidification point and melting range of the suppositories were determined, along with their crushing strength, disintegration time and the time for $80 \%$ of metronidazole to be released from the suppositories ( $\left.t_{80}\right)$.

Results: The ranking of setting time for the suppositories was witepsol H15 > PEG $2850>$ witepsol E75 > PEG 4650, while the ranking of solidification point, melting range, crushing strength, disintegration time and the time for $80 \%$ of metronidazole to be released from the suppositories ( $\left.t_{80}\right)$ was the reverse of that for setting time. Optimal concentrations of Tween 80 and sodium salicylate were observed for the suppository formulations. Using Kitazawa plots, all formulations showed two dissolution rate constants, $k_{1}$ and $k_{2}$ intersecting at time $t_{1}$, with formulations containing 5 to $20 \% \mathrm{w} / \mathrm{w}$ of methylcellulose exhibiting a third dissolution rate constant, $k_{3}$ intersecting with $k_{2}$ at time $t_{2}$.

Conclusion: The physical and release properties of metronidazole suppositories are influenced considerably by the bases and adjuvants employed. Tween 80 and sodium salicylate can probably be used to formulate only immediate-release suppositories while methylcellulose can be useful for sustained-release metronidazole suppositories. Some insight into these inferences can be obtained from parameters derived from Kitazawa plots.
\end{abstract}

Keywords: metronidazole, suppository bases, adjuvants, physical and release properties, Kitazawa plots.

*Corresponding Author: E-mail: oprofit@yahoo.com 


\section{INTRODUCTION}

Suppositories have been recognized as an alternative to the oral route in situations such as when the patient is comatose, unable to swallow or when the drug produces nausea or vomiting. In the light of this, efforts have been made in recent times to present a good number of drugs in suppository form ${ }^{1,2,3,4}$. However, investigators have shown that the physical and release properties of many suppositories depend considerably on the physicochemical properties of the drug, suppository base and formulation adjuvants $^{1,5,6}$ and a lot of formulation work is therefore normally required to optimise the properties of suppository preparations.

In the case of metronidazole, which has also been presented in suppository form ${ }^{2,7,8,9}$ due to various factors, especially gastrointestinal disturbances and nausea when given orally ${ }^{6,7}$, there have been reports of a lot of variability in drug release from different suppository formulations $\mathrm{s}^{2,7}$.

Thus, the present work was designed to investigate the effects of some formulation factors on the physical and release properties of metronidazole suppositories with a view to providing more information on the way to optimize the properties of the resulting suppositories. It was considered of interest to study the effects of different formulation adjuvants with different properties on the physical and release properties of metronidazole in witepsol and polyethylene glycol (PEG) suppository formulations.

\section{MATERIALS AND METHODS Materials}

The materials used were metronidazole BP (Rhone Poulenc, France); Witepsol H15 and E75 (Huls AG, France); Polyethylene glycol, PEG 1500 and PEG 6000 (Merck, Germany); Tween 80 and sodium salicylate (Merck, Germany); and methylcellulose (Bufa B.V., Netherlands).

\section{Preparation of suppositories}

Suppositories $(1 \mathrm{~g})$ containing $200 \mathrm{mg}$ of metronidazole each were prepared by the fusion method following a previously described method ${ }^{2}$ using Witepsol H15, Witepsol E75, and blends of polyethylene glycol 1500 and $6000 \quad$ (70\%:30\% and 30\%:70\%) to give PEG 2850 and PEG 4650, respectively. The appropriate quantity of each suppository base was melted on a water bath. Prior to incorporation, metronidazole powder was passed through a $100 \mu \mathrm{m}$ mesh sieve. Sodium salicylate was micronised and also passed through the same sieve. Quantities corresponding to $200 \mathrm{mg}$ of the metronidazole powder with and without adjuvant were added with trituration, using displacement value calculations. Adjuvants (Tween 80 , sodium salicylate or methylcellulose) were added in different concentrations of $1,3,5$ or $10 \% \mathrm{w} / \mathrm{w}$ for Tween 80 or sodium salicylate, and 1,5,10 or $20 \% \mathrm{w} / \mathrm{w}$ for methylcellulose. Mixing was continued until a homogeneous mass was produced. The mass was poured into stainless steel moulds, allowed to cool and the suppositories removed. Suppositories were weighed and kept at room temperature for $24 \mathrm{~h}$ after removal from the mould to allow for uniform solidification and crystal transformation. The prepared suppositories were wrapped in aluminium foil and stored in a dessicator in a refrigerator until needed.

\section{Evaluation of physical properties of suppositories}

The prepared suppositories were subjected to several physical tests. The ease of moulding of the suppositories was assessed by the setting time of the molten mass, which was taken as the time for the suppository to set in the mould ${ }^{10}$.

Solidification point was also determined ${ }^{10}$. The test suppository was melted in a test tube at $45^{\circ} \mathrm{C}$. A thermometer was dipped inside the cooling mass and rotated mechanically. The temperature at which the mass first began to adhere to the thermometer was recorded as the solidification point ${ }^{10}$. 
Melting range was determined using the ascending melting point method $^{10}$. Suppositories to be tested were melted in a water bath. One end of a straight capillary tube was dipped into the molten mass. The molten mass was allowed to rise to $5 \mathrm{~cm}$ in the capillary tube and stored for $24 \mathrm{~h}$ in the refrigerator. The capillary tube was then attached to a thermometer graduated in 0.20 increments and lowered into a water bath heated so that the temperature rose at the rate of $1^{\circ} \mathrm{C}$ per minute. The temperature at which the suppository began to melt and the temperature when clarification was observed were recorded as a range for the melting point.

The prepared suppositories were evaluated for uniformity of weight using twenty (20) suppositories for each formulation in accordance with the BP method ${ }^{11}$. Content uniformity was determined by spectrophotometric method. The suppositories were individually melted and dissolved in $100 \mathrm{ml}$ of phosphate buffer. After dilution, the absorbance was measured on a spectrophotometer (Pye Unicam, SP 6-550, Cambridge, England) at a wavelength of $275 \mathrm{~nm}$. It was established in preliminary experiments that the suppository bases and other materials did not interfere with absorbance measurement of metronidazole. The Erweka breaking strength tester (Erweka Apparatebau $\mathrm{GmbH}$, Germany) was used to measure the resistance of the suppositories to crushing. All determinations were done in quadruplicate.

\section{Evaluation of disintegration and release properties of the suppositories}

Disintegration time of the suppositories was determined $^{2}$. The suppository to be tested was placed in a special glass container with perforated ends and immersed in a water bath maintained at $37 \pm 1^{\circ} \mathrm{C}$. A magnetic stirrer set at $100 \mathrm{rpm}$ was placed in the glass container. The time for disintegration was noted when the suppository has completely melted (Witepsol) or dissolved (PEG) in the medium.
In vitro release tests were carried out according to the USP XX basket method using the Hanson Easi-Lift dissolution equipment. Each suppository was placed in the basket ${ }^{12}$ and lowered into a flask containing $500 \mathrm{ml}$ of

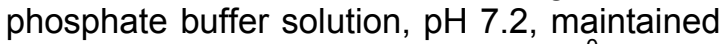
at a constant temperature $\left(37 \pm 0.5^{\circ} \mathrm{C}\right)$. The basket was rotated at a constant speed of 50rpm. Samples $(5 \mathrm{ml})$ were withdrawn at specified time intervals. Fresh buffer solution maintained at experimental temperature was used to replace the same volume of withdrawn samples.The amount of metronidazole in each sample was analysed spectophotometrically at $275 \mathrm{~nm}$ (Pye Unicam, SP 6-550, Cambridge, England). The mean of four determinations was used to calculate drug release from each of the formulation.

The integrated form of the equation of Noyes and Whitney ${ }^{13}$, which has found wide application in describing the release kinetics of drugs from tablet dosage forms ${ }^{14,15,16}$ is given below:

$\ln \left[\mathrm{C}_{\mathrm{s}} /\left(\mathrm{C}_{\mathrm{s}}-\mathrm{C}\right)\right] \quad=\quad \mathrm{kt}$

Where $C_{s}$ is the concentration of the solute at saturation, $C$ is its concentration at time $t$, and $\mathrm{k}$ is a dissolution rate constant. Adegboye and Itiola ${ }^{2}$ have found this equation applicable to the release kinetics of suppository

formulations. Kitazawa plots of $\ln [\mathrm{Cs} /(\mathrm{Cs}-\mathrm{C})]$ versus $t$ were constructed for all formulations ${ }^{14}$.

\section{Statistical analysis}

Results are expressed as the mean \pm SD of four determinations. Analysis of variance (ANOVA) was used to test the statistical significance of differences among groups.

\section{RESULTS}

The results of all physical tests are presented in Table 1. The results show that all prepared suppositories satisfied the $\mathrm{BP}$ requirement ${ }^{11}$ for weight uniformity, with none of the individual weights deviating from the average by more than $5 \%$. Also, the mean drug content for the suppositories was found to satisfy the $B P$ requirement ${ }^{11}$ for content uniformity for most suppository formulations. 


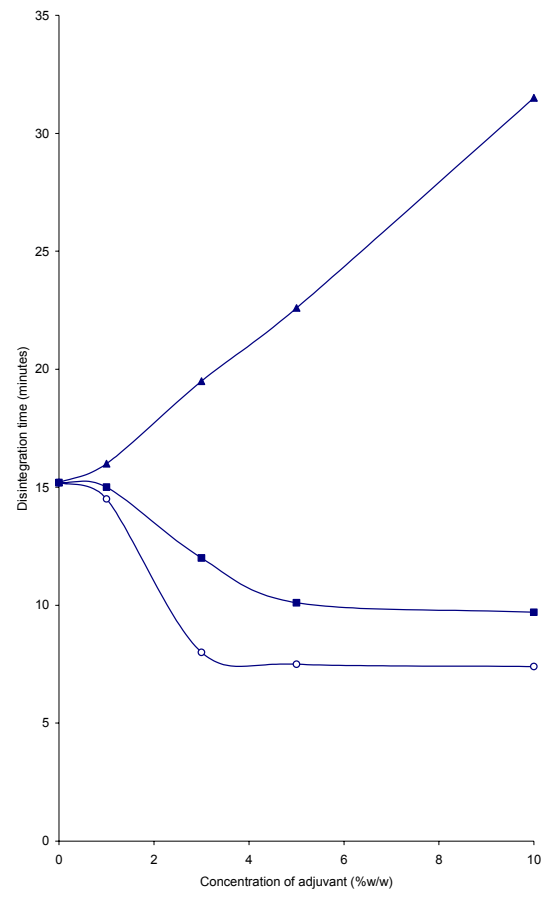

Figure 1: Plots of disintegration time against concentration of adjuvant for witepsol $\mathrm{H} 15$ formulations containing different adjuvants. ○- Tween 80 ; - sodium salicylate;

$\Delta$ - methylcellulose
Itiola \& Adegboye

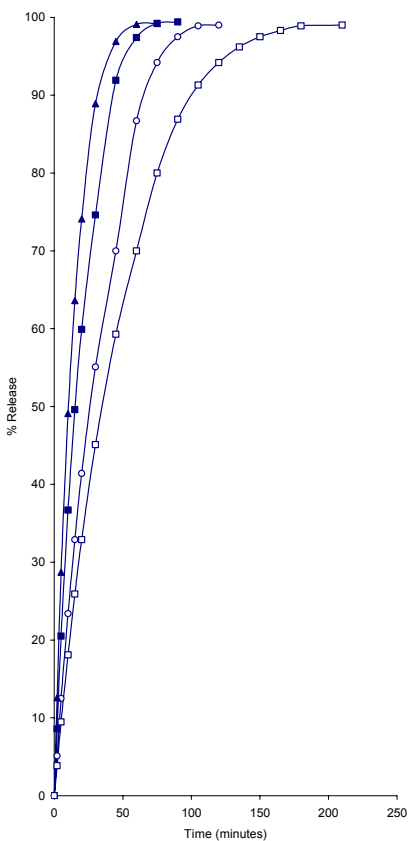

Figure 2: Plots of release profiles of metronidazole from witepsol E75 containing $5 \% \mathrm{w} / \mathrm{w}$ of the different adjuvants. $\circ$ - blank; $\boldsymbol{\Delta}$ - Tween 80 ; sodium salicylate; $\square-$ methylcellulose.

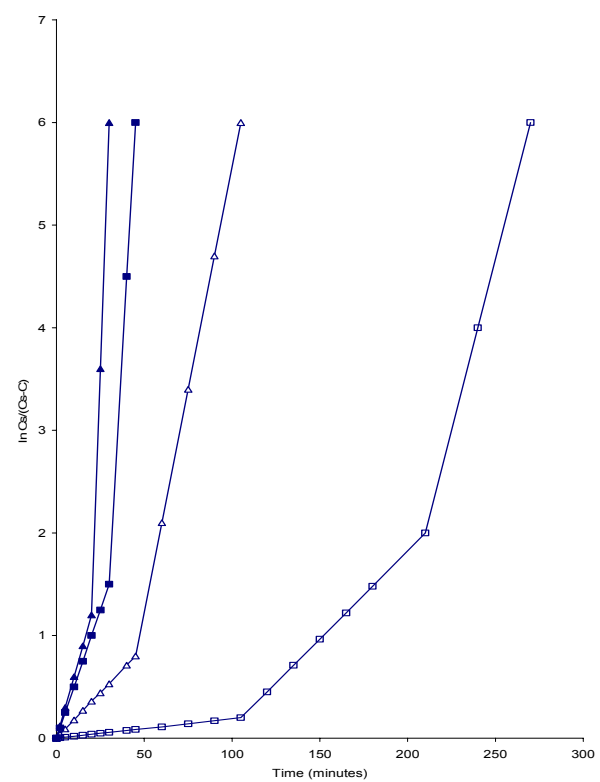

Figure 3: Kitazawa plots of release of metronidazole from PEG 4650 formulations containing $5 \% \mathrm{w} / \mathrm{w}$ of the different adjuvants. $\Delta$ - blank; $\mathbf{-}$ - Tween $80 ; \boldsymbol{\Delta}$ - sodium salicylate; $\square$ methylcellulose.

None of the suppository formulations had less than $90 \%$ and none had more than $110 \%$ of

expected metronidazole content. 
Itiola \& Adegboye

Table 1: Physicochemical properties of metronidazole suppositories

\begin{tabular}{|c|c|c|c|c|c|c|c|c|}
\hline Base & Adjuvant & $\begin{array}{c}\text { Conc. } \\
\text { of } \\
\text { adjuvan } \\
t\end{array}$ & $\begin{array}{c}\text { Setting } \\
\text { time (min) } \\
\text { S(SD) }\end{array}$ & $\begin{array}{c}\text { Weight(g) } \\
\text { X(SD) }\end{array}$ & $\begin{array}{c}\text { Drug } \\
\text { content } \\
\%(S D)\end{array}$ & $\begin{array}{c}\text { Solidificatio } \\
\text { n point } \\
{ }^{0} \mathrm{C}(\mathrm{SD})\end{array}$ & $\begin{array}{l}\text { Melting } \\
\text { range }^{\circ} \mathrm{C}\end{array}$ & $\begin{array}{c}\text { Crushin } \\
\text { g } \\
\text { strength } \\
\mathrm{N} \text { (SD) }\end{array}$ \\
\hline \multirow[t]{4}{*}{$\begin{array}{l}\text { Witepso } \\
\text { I H15 }\end{array}$} & - & 0.0 & $17.50(0.12)$ & $1.010(0.020)$ & $98.4(2.4)$ & $30.0(0.4)$ & $\begin{array}{l}33.5- \\
35.0\end{array}$ & $14.7(1.0)$ \\
\hline & Tween 80 & $\begin{array}{c}1.0 \\
3.0 \\
5.0 \\
10.0\end{array}$ & $\begin{array}{l}17.15(0.24) \\
14.80(1.44) \\
14.00(1.12) \\
13.55(1.32)\end{array}$ & $\begin{array}{l}1.014(0.040) \\
1.018(0.080) \\
1.025(0.024) \\
1.040(0.050)\end{array}$ & $\begin{array}{l}96.2(1.8) \\
96.9(1.5) \\
98.4(0.8) \\
98.2(2.3)\end{array}$ & $\begin{array}{l}30.0(1.9) \\
29.5(2.2) \\
29.0(1.9) \\
28.5(0.5)\end{array}$ & $\begin{array}{c}33.5- \\
35.0 \\
32.5- \\
34.0 \\
31.5- \\
32.5 \\
30.0- \\
31.5\end{array}$ & $\begin{array}{l}15.1(1.6) \\
15.7(2.7) \\
16.7(2.1) \\
16.9(1.4)\end{array}$ \\
\hline & $\begin{array}{l}\text { Sodium } \\
\text { salicylate }\end{array}$ & $\begin{array}{c}1.0 \\
3.0 \\
5.0 \\
10.0\end{array}$ & $\begin{array}{c}15.00(0.24) \\
13.86(1.44) \\
9.60(1.12) \\
8.50(1.32)\end{array}$ & $\begin{array}{l}1.011(0.034) \\
1.014(0.018) \\
1.019(0.024) \\
1.028(0.015)\end{array}$ & $\begin{array}{l}96.2(1.8) \\
96.9(1.5) \\
98.4(0.8) \\
98.2(2.3)\end{array}$ & $\begin{array}{l}32.0(1.9) \\
32.5(2.2) \\
33.5(0.9) \\
34.5(1.5)\end{array}$ & $\begin{array}{c}33.5- \\
35.5 \\
33.5- \\
36.0 \\
33.5- \\
36.5 \\
33.5- \\
37.0\end{array}$ & $\begin{array}{l}16.7(1.3) \\
19.6(2.4) \\
21.5(0.1) \\
22.2(1.2)\end{array}$ \\
\hline & Methylcellulose & $\begin{array}{c}1.0 \\
5.0 \\
10.0 \\
20.0\end{array}$ & $\begin{array}{c}13.50(0.24) \\
10.10(1.44) \\
6.40(1.12) \\
5.80(1.32)\end{array}$ & $\begin{array}{l}1.019(0.017) \\
1.024(0.080) \\
1.033(0.024) \\
1.056(0.005)\end{array}$ & $\begin{array}{l}96.2(1.8) \\
96.9(1.5) \\
98.4(0.8) \\
98.2(2.3)\end{array}$ & $\begin{array}{l}32.5(1.9) \\
33.0(1.3) \\
34.5(1.8) \\
35.5(1.6)\end{array}$ & $\begin{array}{c}33.5- \\
36.0 \\
34.0- \\
36.0 \\
34.5- \\
37.0 \\
35.0- \\
38.0\end{array}$ & $\begin{array}{l}18.6(1.0) \\
24.9(2.7) \\
28.4(2.1) \\
32.0(0.8)\end{array}$ \\
\hline \multirow[t]{4}{*}{$\begin{array}{l}\text { Witepso } \\
\text { I E75 }\end{array}$} & - & 0.0 & $10.55(0.12)$ & $1.060(0.020)$ & $97.6(1.3)$ & $34.0(1.1)$ & $\begin{array}{c}36.5- \\
38.0\end{array}$ & $21.6(2.0)$ \\
\hline & Tween 80 & $\begin{array}{c}1.0 \\
3.0 \\
5.0 \\
10.0\end{array}$ & $\begin{array}{l}10.00(0.24) \\
8.50(1.44) \\
7.75(1.12) \\
7.20(1.32)\end{array}$ & $\begin{array}{l}1.066(0.045) \\
1.070(0.037) \\
1.077(0.024) \\
1.080(0.019)\end{array}$ & $\begin{array}{l}96.2(1.8) \\
96.9(1.5) \\
98.4(0.8) \\
98.2(2.3)\end{array}$ & $\begin{array}{l}34.0(2.7) \\
33.5(1.1) \\
33.0(1.4) \\
32.5(1.2)\end{array}$ & $\begin{array}{c}36.5- \\
38.0 \\
36.0- \\
37.0 \\
35.0- \\
36.0 \\
34.5- \\
35.0\end{array}$ & $\begin{array}{l}22.7(2.2) \\
25.5(2.3) \\
26.3(0.9) \\
27.4(1.0)\end{array}$ \\
\hline & $\begin{array}{l}\text { Sodium } \\
\text { salicylate }\end{array}$ & $\begin{array}{c}1.0 \\
3.0 \\
5.0 \\
10.0\end{array}$ & $\begin{array}{l}9.00(0.24) \\
8.10(1.44) \\
6.00(1.12) \\
5.40(1.32)\end{array}$ & $\begin{array}{l}1.062(0.012) \\
1.065(0.016) \\
1.069(0.028) \\
1.075(0.031)\end{array}$ & $\begin{array}{l}96.2(1.8) \\
96.9(1.5) \\
98.4(0.8) \\
98.2(2.3)\end{array}$ & $\begin{array}{l}35.5(1.8) \\
36.5(0.9) \\
37.0(1.1) \\
38.0(1.1)\end{array}$ & $\begin{array}{c}36.5- \\
38.5 \\
36.5- \\
39.0 \\
36.5- \\
39.5 \\
36.5- \\
40.0\end{array}$ & $\begin{array}{l}28.0(1.4) \\
30.8(2.3) \\
31.6(1.7) \\
32.5(0.4)\end{array}$ \\
\hline & Methylcellulose & $\begin{array}{c}1.0 \\
5.0 \\
10.0 \\
20.0\end{array}$ & $\begin{array}{l}7.00(0.24) \\
5.80(1.44) \\
4.40(1.12) \\
3.00(1.32)\end{array}$ & $\begin{array}{l}1.071(0.019) \\
1.078(0.027) \\
1.085(0.025) \\
1.096(0.005)\end{array}$ & $\begin{array}{l}96.2(1.8) \\
96.9(1.5) \\
98.4(0.8) \\
98.2(2.3)\end{array}$ & $\begin{array}{l}36.0(1.3) \\
37.0(1.2) \\
37.5(1.7) \\
38.5(0.5)\end{array}$ & $\begin{array}{c}37.0- \\
38.5 \\
37.5- \\
39.0 \\
37.5- \\
39.5 \\
38.0- \\
40.0\end{array}$ & $\begin{array}{l}29.4(0.1) \\
34.5(0.6) \\
37.2(1.6) \\
41.1(0.9)\end{array}$ \\
\hline
\end{tabular}


Itiola \& Adegboye

\begin{tabular}{|c|c|c|c|c|c|c|c|c|}
\hline \multirow[t]{4}{*}{$\begin{array}{l}\text { OPEG } \\
2850\end{array}$} & - & 0.0 & $14.70(0.12)$ & $1.040(0.020)$ & $98.4(2.4)$ & $31.0(0.4)$ & $\begin{array}{l}35.0- \\
37.0\end{array}$ & $17.6(0.5)$ \\
\hline & Tween 80 & $\begin{array}{c}1.0 \\
3.0 \\
5.0 \\
10.0\end{array}$ & $\begin{array}{l}14.00(0.24) \\
12.90(1.44) \\
11.00(1.12) \\
10.50(1.32)\end{array}$ & $\begin{array}{l}1.045(0.240) \\
1.048(0.180) \\
1.054(0.024) \\
1.060(0.005)\end{array}$ & $\begin{array}{l}96.2(1.8) \\
96.9(1.5) \\
98.4(0.8) \\
98.2(2.3)\end{array}$ & $\begin{array}{l}31.5(1.9) \\
31.5(1.2) \\
32.0(1.6) \\
32.5(1.5)\end{array}$ & $\begin{array}{c}35.0- \\
37.0 \\
34.5- \\
36.0 \\
34.0- \\
35.5 \\
33.5- \\
35.0\end{array}$ & $\begin{array}{l}18.6(0.7) \\
20.5(1.6) \\
22.5(1.4) \\
25.4(1.2)\end{array}$ \\
\hline & $\begin{array}{l}\text { Sodium } \\
\text { salicylate }\end{array}$ & $\begin{array}{c}1.0 \\
3.0 \\
5.0 \\
10.0\end{array}$ & $\begin{array}{l}12.00(0.24) \\
6.42(1.44) \\
6.00(1.12) \\
5.20(1.32)\end{array}$ & $\begin{array}{l}1.041(0.240) \\
1.043(0.180) \\
1.046(0.024) \\
1.050(0.005\end{array}$ & $\begin{array}{l}96.2(1.8) \\
96.9(1.5) \\
98.4(0.8) \\
98.2(2.3)\end{array}$ & $\begin{array}{l}31.5(1.9) \\
32.0(1.1) \\
33.0(0.3) \\
34.0(1.4)\end{array}$ & $\begin{array}{c}33.5- \\
36.5 \\
36.0- \\
37.5 \\
36.5- \\
38.0 \\
36.0- \\
38.5\end{array}$ & $\begin{array}{l}19.0(2.0) \\
21.5(1.9) \\
25.5(0.2) \\
30.4(0.8)\end{array}$ \\
\hline & $\begin{array}{l}\text { methylcellulos } \\
\mathrm{e}\end{array}$ & $\begin{array}{c}1.0 \\
5.0 \\
10.0 \\
20.0\end{array}$ & $\begin{array}{c}10.00(0.24) \\
8.50(1.44) \\
6.25(1.12) \\
5.00(1.32)\end{array}$ & $\begin{array}{c}1.048(0.240) \\
1.055(0.180) \\
1.065(0.024) \\
1.080(0.005\end{array}$ & $\begin{array}{l}96.2(1.8) \\
96.9(1.5) \\
98.4(0.8) \\
98.2(2.3)\end{array}$ & $\begin{array}{l}32.0(1.3) \\
33.5(1.2) \\
34.0(1.5) \\
35.0(1.5)\end{array}$ & $\begin{array}{c}35.5- \\
37.0 \\
36.5- \\
38.0 \\
37.0- \\
39.0 \\
37.5- \\
39.0\end{array}$ & $\begin{array}{l}21.6(0.6) \\
25.5(2.2) \\
29.4(1.1) \\
37.2(1.0)\end{array}$ \\
\hline \multirow[t]{4}{*}{$\begin{array}{l}\text { PEG } \\
4650\end{array}$} & - & 0.0 & $7.40(0.12$ & $1.090(0.020$ & $97.6(1.3)$ & $34.5(2.0)$ & $\begin{array}{l}38.0- \\
40.0\end{array}$ & $24.5(0.1)$ \\
\hline & Tween 80 & $\begin{array}{c}1.0 \\
3.0 \\
5.0 \\
10.0\end{array}$ & $\begin{array}{l}7.20(0.24) \\
6.00(1.44) \\
4.80(1.12) \\
4.10(1.32)\end{array}$ & $\begin{array}{c}1.093(0.240) \\
1.097(0.180) \\
1.099(0.024) \\
1.103(0.005\end{array}$ & $\begin{array}{l}96.2(1.8) \\
96.9(1.5) \\
98.4(0.8) \\
98.2(2.3)\end{array}$ & $\begin{array}{l}34.5(1.3) \\
35.0(1.0) \\
35.5(1.6) \\
36.0(1.5)\end{array}$ & $\begin{array}{c}38.0- \\
39.5 \\
37.5- \\
39.0 \\
37.0- \\
38.5 \\
36.5- \\
38.0\end{array}$ & $\begin{array}{l}25.4(1.2) \\
26.5(0.2) \\
28.4(0.5) \\
30.3(0.8)\end{array}$ \\
\hline & $\begin{array}{l}\text { Sodium } \\
\text { salicylate }\end{array}$ & $\begin{array}{c}1.0 \\
3.0 \\
5.0 \\
10.0\end{array}$ & $\begin{array}{l}6.50(0.24) \\
4.40(1.44) \\
4.00(1.12) \\
3.50(1.32)\end{array}$ & $\begin{array}{c}1.091(0.240) \\
1.093(0.180) \\
1.095(0.024) \\
1.099(0.005\end{array}$ & $\begin{array}{l}96.2(1.8) \\
96.9(1.5) \\
98.4(0.8) \\
98.2(2.3)\end{array}$ & $\begin{array}{l}35.0(1.0) \\
35.5(1.2) \\
36.0(0.1) \\
37.0(2.5)\end{array}$ & $\begin{array}{c}38.0- \\
40.0 \\
38.0- \\
41.0 \\
38.0- \\
41.5 \\
38.0- \\
42.0\end{array}$ & $\begin{array}{l}26.5(2.5) \\
27.5(0.7) \\
29.4(1.3) \\
33.3(0.9)\end{array}$ \\
\hline & $\begin{array}{l}\text { methylcellulos } \\
\mathrm{e}\end{array}$ & $\begin{array}{c}1.0 \\
5.0 \\
10.0 \\
20.0\end{array}$ & $\begin{array}{l}5.00(0.24) \\
4.00(1.44) \\
2.80(1.12) \\
2.00(1.32)\end{array}$ & $\begin{array}{l}1.095(0.240) \\
1.099(0.180) \\
1.103(0.024) \\
1.110(0.005)\end{array}$ & $\begin{array}{l}96.2(1.8) \\
96.9(1.5) \\
98.4(0.8) \\
98.2(2.3)\end{array}$ & $\begin{array}{l}35.5(2.2) \\
36.5(1.2) \\
37.5(1.8) \\
39.0(0.5)\end{array}$ & $\begin{array}{c}38.0- \\
40.5 \\
39.0- \\
41.0 \\
39.5- \\
41.5 \\
40.0- \\
42.0\end{array}$ & $\begin{array}{l}27.4(1.0) \\
32.3(2.0) \\
38.2(1.5) \\
44.1(1.8)\end{array}$ \\
\hline
\end{tabular}


From Table 1, it is also seen that the ranking of setting time, which indicates the ease of moulding, for the different bases was witepsol H15 > PEG 2850 > witepsol E75 > PEG 4650. It is notable however that the ranking of solidification point, melting range and crushing strength for the different bases was a reverse of the ranking for setting time: PEG $4650>$ witepsol E75 > PEG 2850 > witepsol H15. Table 1 also shows that the adjuvants exhibited considerable effects on the different parameters. Setting time decreased with incorporation of all the adjuvants while crushing strength increased. On the other hand, values of melting range and solidification point were decreased by incorporation of Tween 80, but increased with methylcellulose and sodium salicylate.

Figure 1 shows representative plots of disintegration time $\left(D_{T}\right)$ against concentration of adjuvant. Figure 2 shows representative release profiles of metronidazole from witepsol E75 containing $5 \% \mathrm{w} / \mathrm{w}$ of the different adjuvants. The time for $80 \%$ of metronidazole to be released $\left(t_{80}\right)$ was obtained for all formulations. The values of $D_{T}$ and $t_{80}$ are presented in Table 2, and both parameters also show ranking for the different bases which was the reverse of that for setting time: PEG 4650 > witepsol E75 > PEG $2850>$ witepsol $H 15$. The values of $D_{T}$ and $t_{80}$ decreased with increasing concentration of Tween 80 and sodium salicylate, but increased with methylcellulose.

Figure 3 shows representative Kitazawa plots of release of metronidazole from PEG 4650 formulations containing $5 \% \mathrm{w} / \mathrm{w}$ of the different adjuvants. Two linear regression lines of slopes $k_{1}$ and $k_{2}$ intersecting at time $t_{1}$ were obtained for all formulations, while an additional third regression line of slope $k_{3}$ intersecting at time $t_{2}$ was obtained for formulations containing 5,10 and $20 \% \mathrm{w} / \mathrm{w}$ methylcellulose. Values of all parameters derived from Kitazawa plots are included in Table 2. It is seen that the values of $k_{1}$ and $k_{2}$ increased with increased in concentration of Tween 80 or sodium salicylate, but decreased with increase in concentration of methylcellulose. The values of $k_{3}$ also decreased with increasing concentration of methylcellulose for the relevant suppository formulations.

\section{DISCUSSION}

The physical and release properties of metronidazole suppositories were studied to provide more insights into the ways of optimizing the rectal formulation of the drug. The results obtained indicate that the prepared suppositories met official standards with regard to weight and content uniformity as presented in Table 1. The results (Tables 1 and 2) also show that the physical and release properties of the suppositories were affected considerably by the formulation variables employed. The rankings of most of the physical and release parameters for the different suppository bases show that the bases can probably be employed to formulate metronidazole suppository formulations with a variety of physical and release properties for different purposes. The rankings also suggest that the values of most of the parameters increased with the ease with which the suppositories were moulded in the different bases as measured by the setting time.

The ranking of the dissolution rate constants was $\mathrm{k}_{1}<\mathrm{k}_{2}<\mathrm{k}_{3}$ for relevant formulations (Table 2), showing an increase in the rate of release of metronidazole with time. The $t_{1}$ values were found to decrease with increase in concentration of Tween 80 and sodium salicylate, but increased with increase in concentration of methylcellulose. The $t_{2}$ values (for formulations containing 5,10 and $20 \% \mathrm{w} / \mathrm{w}$ methylcellulose) also increased with increase in methylcellulose content. Table 2 also shows that for all formulations, time $t_{1}$ was generally greater than the disintegration time, $D_{T}$. Thus the disintegration time for the formulations did not correspond with the time for the change from $k_{1}$ to $k_{2}$ at $t_{1}$, but rather $D_{T}$ occurred before $t_{1}$. While this may be attributed to the greater agitation associated with the disintegration test ${ }^{15}$, it may also 
Itiola \& Adegboye

Table 2: Release properties of metronidazole suppositories

\begin{tabular}{|c|c|c|c|c|c|c|c|c|c|}
\hline Base & Adjuvant & $\begin{array}{c}\text { Conc. of } \\
\text { adjuvan } \\
t\end{array}$ & $\begin{array}{c}\mathrm{D}_{\mathrm{T}} \\
\text { (min) }\end{array}$ & $t_{80}(\min )$ & $k_{1}$ & $\mathrm{k}_{2}$ & $\mathbf{k}_{3}$ & $\begin{array}{c}t_{1} \\
(\mathrm{~min})\end{array}$ & $\begin{array}{c}\mathrm{t}_{2} \\
(\mathrm{~min})\end{array}$ \\
\hline \multirow{13}{*}{$\begin{array}{l}\text { Witepsol } \\
\text { H15 }\end{array}$} & - & 0.0 & $15.20(1.11)$ & $23.60(0.56)$ & 0.067 & 0.081 & - & 20 & - \\
\hline & \multirow[t]{4}{*}{ Tween 80} & 1.0 & $14.42(1.56)$ & $16.58(1.21)$ & 0.090 & 0.210 & - & 15 & - \\
\hline & & 3.0 & $7.85(1.48)$ & $11.15(0.04)$ & 0.135 & 0.315 & - & 10 & - \\
\hline & & 5.0 & $7.53(2.36)$ & $10.90(1.71)$ & 0.137 & 0.333 & - & 10 & - \\
\hline & & 10.0 & $7.41(2.56)$ & 10.82(0.84) & 0.140 & 0.350 & - & 10 & - \\
\hline & Sodium & 1.0 & $15.00(1.56)$ & $22.80(0.22)$ & 0.069 & 0.104 & - & 20 & - \\
\hline & \multirow[t]{3}{*}{ salicylate } & 3.0 & $14.80(1.48)$ & $22.00(0.90)$ & 0.072 & 0.122 & - & 20 & - \\
\hline & & 5.0 & $10.15(2.36)$ & $16.45(1.06)$ & 0.093 & 0.206 & - & 15 & - \\
\hline & & 10.0 & $9.56(2.56)$ & $16.24(0.34)$ & 0.096 & 0.216 & - & 15 & - \\
\hline & \multirow[t]{4}{*}{ Methylcellulose } & 1.0 & $16.00(1.56)$ & $36.56(0.65)$ & 0.045 & 0.060 & - & 30 & - \\
\hline & & 5.0 & $19.50(1.48)$ & $53.30(1.11)$ & 0.030 & 0.042 & 0.066 & 45 & 75 \\
\hline & & 10.0 & $22.64(2.36)$ & $70.00(0.70)$ & 0.015 & 0.032 & 0.043 & 60 & 90 \\
\hline & & 20.0 & $31.50(2.56)$ & $88.68(0.04)$ & 0.007 & 0.021 & 0.035 & 75 & 150 \\
\hline Witepsol & - & 0.0 & $28.60(1.11)$ & $53.65(0.54)$ & 0.026 & 0.055 & - & 45 & - \\
\hline \multirow[t]{12}{*}{ E75 } & \multirow[t]{4}{*}{ Tween 80} & 1.0 & $27.00(1.56)$ & $37.90(0.78)$ & 0.040 & 0.060 & - & 30 & - \\
\hline & & 3.0 & $20.65(1.48)$ & $24.40(1.12)$ & 0.065 & 0.082 & - & 20 & - \\
\hline & & 5.0 & $19.44(2.36)$ & $24.00(0.92)$ & 0.067 & 0.085 & - & 20 & - \\
\hline & & 10.0 & $19.25(2.56)$ & $23.70(1.40)$ & 0.070 & 0.087 & - & 20 & - \\
\hline & Sodium & 1.0 & $28.00(1.56)$ & $50.55(1.36)$ & 0.030 & 0.057 & - & 45 & - \\
\hline & \multirow[t]{3}{*}{ salicylate } & 3.0 & $27.48(1.48)$ & $48.68(1.78)$ & 0.032 & 0.061 & - & 45 & - \\
\hline & & 5.0 & $20.72(2.36)$ & $34.00(0.06)$ & 0.045 & 0.076 & - & 30 & - \\
\hline & & 10.0 & $19.58(2.56)$ & $33.80(0.50)$ & 0.046 & 0.078 & - & 30 & - \\
\hline & \multirow[t]{4}{*}{ Methylcellulose } & 1.0 & $29.00(1.56)$ & $67.55(0.99)$ & 0.022 & 0.042 & - & 60 & - \\
\hline & & 5.0 & $32.40(1.48)$ & $75.00(0.22)$ & 0.020 & 0.027 & 0.053 & 60 & 75 \\
\hline & & 10.0 & $36.16(2.36)$ & $93.00(1.00)$ & 0.016 & 0.019 & 0.041 & 75 & 105 \\
\hline & & 20.0 & $41.00(2.56)$ & $136.00(0.21)$ & 0.013 & 0.008 & 0.028 & 90 & 180 \\
\hline PEG & - & 0.0 & $20.88(1.11)$ & $38.55(0.88)$ & 0.042 & 0.055 & - & 30 & - \\
\hline \multirow[t]{12}{*}{2850} & \multirow{4}{*}{ Tween 80} & 1.0 & $19.25(1.56)$ & $34.68(0.58)$ & 0.046 & 0.062 & - & 30 & - \\
\hline & & 3.0 & $15.82(1.48)$ & $24.00(0.96)$ & 0.066 & 0.079 & - & 20 & - \\
\hline & & 5.0 & $12.56(2.36)$ & $23.35(1.24)$ & 0.069 & 0.082 & - & 20 & - \\
\hline & & 10.0 & $13.98(2.56)$ & $25.36(0.95)$ & 0.064 & 0.068 & - & 20 & - \\
\hline & Sodium & 1.0 & $20.00(1.56)$ & $30.20(0.61)$ & 0.053 & 0.064 & - & 30 & - \\
\hline & \multirow[t]{3}{*}{ salicylate } & 3.0 & $14.95(1.48)$ & $17.20(0.72)$ & 0.086 & 0.113 & - & 15 & - \\
\hline & & 5.0 & $14.40(2.36)$ & $16.95(0.30)$ & 0.093 & 0.115 & - & 15 & - \\
\hline & & 10.0 & $13.85(2.56)$ & $16.65(0.48)$ & 0.093 & 0.117 & - & 15 & - \\
\hline & \multirow[t]{4}{*}{ methylcellulose } & 1.0 & $22.00(1.56)$ & $50.00(0.55)$ & 0.032 & 0.061 & - & 30 & - \\
\hline & & 5.0 & $25.45(1.48)$ & $57.20(0.81)$ & 0.027 & 0.040 & 0.053 & 45 & 75 \\
\hline & & 10.0 & $29.24(2.36)$ & $71.56(0.76)$ & 0.013 & 0.028 & 0.042 & 60 & 105 \\
\hline & & 20.0 & $34.65(2.56)$ & $96.80(0.88)$ & 0.006 & 0.019 & 0.028 & 75 & 165 \\
\hline \multirow{13}{*}{$\begin{array}{l}\text { PEG } \\
4650\end{array}$} & - & 0.0 & $29.54(2.11)$ & $69.45(1.14)$ & 0.019 & 0.055 & - & 60 & - \\
\hline & \multirow[t]{4}{*}{ Tween 80} & 1.0 & $27.00(1.56)$ & $64.40(1.32)$ & 0.023 & 0.068 & - & 60 & - \\
\hline & & 3.0 & $23.45(1.48)$ & $35.25(0.16)$ & 0.043 & 0.071 & - & 30 & - \\
\hline & & 5.0 & $19.58(2.36)$ & $33.68(0.96)$ & 0.046 & 0.075 & - & 30 & - \\
\hline & & 10.0 & $20.64(2.56)$ & $36.00(0.48)$ & 0.041 & 0.074 & - & 30 & - \\
\hline & Sodium & 1.0 & $28.00(1.56)$ & $53.20(0.64)$ & 0.026 & 0.062 & - & 45 & - \\
\hline & \multirow{3}{*}{ salicylate } & 3.0 & $23.10(1.48)$ & $35.52(1.14)$ & 0.045 & 0.072 & - & 30 & - \\
\hline & & 5.0 & $22.56(2.36)$ & $33.65(0.80)$ & 0.046 & 0.075 & - & 30 & - \\
\hline & & 10.0 & $21.25(2.56)$ & $32.56(0.16)$ & 0.049 & 0.078 & - & 30 & - \\
\hline & \multirow{4}{*}{ methylcellulose } & 1.0 & $31.00(1.56)$ & $87.90(0.18)$ & 0.016 & 0.036 & - & 60 & - \\
\hline & & 5.0 & $34.68(1.48)$ & $104.10(0.46)$ & 0.015 & 0.020 & 0.028 & 60 & 90 \\
\hline & & 10.0 & $40.40(2.36)$ & $123.45(0.44)$ & 0.007 & 0.010 & 0.016 & 75 & 135 \\
\hline & & 20.0 & $46.60(2.56)$ & $168.00(0.36)$ & 0.003 & 0.007 & 0.012 & 90 & 240 \\
\hline
\end{tabular}

suggest that the melting/dissolving time of the suppository does not necessarily correspond exactly to the time of a change in dissolution rate. 
The Kitazawa plot has been used successfully to explain the release kinetics of some tablet formulations. These formulations were found to exhibit monophasic ( $\mathrm{k}_{1}$ only) or biphasic $\left(\mathrm{k}_{1}\right.$ and $k_{2}$ ) release kinetics ${ }^{14,15,16}$. However, it is interesting to note that a significant number of metronidazole suppositories in the present work have exhibited triphasic $\left(\mathrm{k}_{1}, \mathrm{k}_{2}\right.$ and $\left.\mathrm{k}_{3}\right)$ release kinetics. This suggests that release from these suppositories was more complex and involved more processes than release from those tablet formulations. This finding may help to explain some of the variability usually associated with release from suppositories $^{2,7}$. These findings may also be used to manipulate formulation to obtain different types of suppositories for different purposes. This is because it is sometimes preferable to employ sustained-release rather than immediate-release suppositories in therapy. Sustained-release formulations are useful for long term treatment of diseases, such as hypertension, acquired immune deficiency syndrome - AIDS, anaemia, diabetes, and in the treatment of postoperative or malignant pain, especially when the patient is bed-ridden ${ }^{17,18,19}$

The biphasic release rate character of some of the suppositories may be as a result of the release of metronidazole being limited initially by the available surface area of the suppository, while the second (rapid release) phase correspond to the increasing drug release from a melting/dissolving suppository mass. The triphasic release profile of the suppositories containing methylcellulose may be attributed to the ability of methylcellulose to swell and produce a matrix gel structure at certain concentrations ${ }^{15}$. Thus, such suppositories probably maintained their integrity for a longer period to afford an initial slow release of metronidazole, which progressively increased from a slowly melting/dissolving mass.

Both Tween 80 and sodium salicylate increased the release rates of metronidazole from the suppositories. In the case of Tween
80 , this may be due to its ability to improve the wettability of the base matrix and thus enhance diffusion of the embedded drug molecules ${ }^{20}$. The mechanism by which sodium salicylate increased release rate is not clearly understood, but could probably be due to the high solubility of sodium salicylate ${ }^{21,22}$. Rapid leaching of the water soluble sodium salicylate will be expected to introduce porosity and more water sorption into the suppository to promote drug release.

It is important to note that optimum concentrations of adjuvants can be obtained from the present work from the values of most of the physical and release properties studied (Tables 1 and 2) - setting time, crushing strength, disintegration time, $\mathrm{t}_{80}, \mathrm{k}_{1}$ and $\mathrm{k}_{2}$. The values of these parameters were not generally significantly $(\rho>0.05)$ different for PEG formulations containing Tween 80 or sodium salicylate $(\geq 3 \% \mathrm{w} / \mathrm{w})$, and for witepsol formulations containing Tween $80(>3 \% \mathrm{w} / \mathrm{w})$ or sodium salicylate $(>5 \% \mathrm{w} / \mathrm{w})$. These particular concentrations would therefore appear to be optimal for Tween 80 and sodium salicylate in the different formulations. On the other hand, methylcellulose at all concentrations produced significant $(p<0.05)$ effects on all the parameters evaluated.

The rate of release of metronidazole from suppositories containing methylcellulose was much slower than from suppositories containing Tween 80 and sodium salicylate. This was evidenced by the fact that the terminal dissolution rate constant $\left(k_{2}\right)$ values for suppositories containing Tween 80 and sodium salicylate were higher than $k_{2}$ and $k_{3}$ values for formulations containing methylcellulose (Table 2 ). The $t_{1}$ and $t_{2}$ values for relevant methylcellulose formulations were also much higher than $t_{1}$ values for formulations containing Tween 80 and sodium salicylate. Values of $t_{80}$ and $t_{2}$ of up to 168 minutes (2.8 hrs) and 240 minutes (4.0 hrs) respectively (Table 2 ) were obtained for methylcellulose formulations and these suggest that methylcellulose could be useful to formulate sustained-release metronidazole 
suppository formulations, while Tween 80 and sodium salicylate can probably be useful only for immediate-release suppositories.

\section{CONCLUSION}

The study has shown that different physical properties and release rates can be obtained for metronidazole suppositories by using various bases and formulation adjuvants. Optimum concentrations of Tween 80 and sodium salicylate can be employed to formulate immediate-release suppositories. The results also suggest that methylcellulose can be used to formulate sustained-release metronidazole suppositories. Some insights into these inferences on release kinetics of the suppositories can be obtained from parameters derived from Kitazawa plots, and findings from the present work may be exploited in designing a rectal delivery system of metronidazole.

\section{REFERENCES}

1. Zuber, M, Pellion, B, Arnaud, $P$ and Chaumeil, JC. Kinetics of theophyline release from suppositories in vitro: Influence of physicochemical parameters. Int. J. Pharm. 1998; 47: 31-36.

2. Adegboye, TA and Itiola, OA. Formulation effects on the mechanical and release properties of metronidazole suppositories. Afr. J. Med. Med. Sci. 2003; 32: 247-251.

3. Taha, RI, Zaghloul, AA, Samy, AM., Al-Saidan, S, Kassem, AA and Khan, MA. Bioavailiabilty assessment of salbuthamol sulphate suppositories in human volunteers. Int. J. Pharm. 2004; 279: 3-7.

4. Pasztor, E, Csoka, G, Klebovich, I and Antal, I. Formulation study of metolose ${ }^{\circledR}$ based on insitu gelling liquid suppository. Eur. J. Pharm. Sci. 2007; 6: 34-39.

5. Onyeji, CO, Adebayo, AS, and Babalola, CP. Effects of absorption enchancers in chloroquine suppository formulations. 1: In vitro release characteristics. Eur. J. Pharm. Sci. 1999; 9: 131-136.

6. Ozyazia, CM, Turgut, EH., Taner, MS., Koseoglu, K and Ertan, G. In vitro evaluation and vaginal absorption of metronidazole suppositories in rabbits. J. Drug Target. 2003; 3: 177-185.

7. Vromans, HG, Moolenaar, F, Visser, $J$ and Meijer, DK. Rectal absorption of metronidazole from polyethylene glycerol suppositories. Pharm. Weekbl. Sci. 6: 18-20.

8. Steinman, A, Gips, M, Lavy, E, Sinay, I and Soback, S. Pharmacokinetics of metronidazole in horses after intravenous, rectal and oral administration. J Vet. Pharm. Therap, 2000; 6:353-357.

9. Ofoefule, SI, Ibezim, EC, Esimine, OC, Pepple, MN, Njoku, CN and Orisakwe, EO. (2004). Bioavailiability of metronidazole in rabbits after administration of a rectal suppository. Amer. J. Therap. 11; 3:190-193

10. Coben, LJ and Lieberman, HA. 1986. Suppositories. The Theory and Practice of Industrial Pharmacy. L Lachman, H A. Lieberman and JL. Kanig. Eds. $3^{\text {rd }}$ ed. Philadelphia: Lea and Febiger. 564-588

11. British Pharmacopoeia. I\&II. London: Her Majesty's Stationery Office. 1998.

12. United States Pharamcopoeia XX. $1980.20^{\text {th }}$ ed. Easton: Mark Publishing Company. 959.

13. Noyes, AA and Whitney, WR. The rate of dissolution of solid substances in their own solutions. J Amer. Chem. Soc, 1897; 19: 930-934.

14. Kitazawa, S, Johno, I, Ito, $Y$, Teramura, $S$ and Okada, J. Effect of hardness on the disintegration and dissolution rate of uncoated caffeine tablets. J. Pharm. Pharmacol. 1975; 27: 765-770.

15. Itiola, OA. and Pilpel, N. Studies on metronidazole tablet formulations. J. Pharm. Pharmacol. 1986; 38:81-86

16. Odeku, OA and Itiola, OA. Evaluation of the effects of khaya gum on the mechanical and release properties of paracetamol tablets. Drug Dev. Ind. Pharm. 2003; 29 :311-320.

17. Moolenaar, F, Meyler,P, Frijlink, E, Jauw, TH, Visser, $\mathrm{J}$ and Proost, $H$. Rectal absorption of morphine from controlled-release suppositories. Int. J. Pharm. 1995; 114:117-120.

18. Azechi, Y, Ishikawa, K, Mizuno, N, and Takahashi, $K$. Sustained-release of diclofenac from polymercontaining suppositories and the mechanisms involved. Drug Dev. Ind. Pharm. 2000; 26: 1177-1183.

19. Takatori, $Y$, Shimono, $N$, Higaki,K and Kimra, $T$. Evaluation of sustained-release suppositories prepared with fatty base including solid fats with high melting points. Int. J. Pharm. 2004; 278: 275-282.

20. Hanaee, J, Javadzadeh, Y, Taftachi, S, Farid, D and Nokhodchi,A. The role of various surfactants on the release of salbutamol from suppositories. Int. J. Pharm. 2004; 59: 903-906.

21. Nishihata, T, Rytting, TH, Higuchi, $T$ and Caldwell, $L$. Enhancement of rectal absorption of insulin using salicylates in dogs. J. Pharm. Pharmacol. 1983; 35: 148-151.

22. Novak, E, Osborne, DW, Matheson, LK, Parott, EL, Lach, JL and Morrison, WB. Effects of salicylates on rectal absorption of cefmetazole in healthy human volunteers. Drug Dev. Ind. Pharm. 1991; 17: 373 - 389 